\title{
Other Gynecologic Pathology in Endometrial Cancer Patients
}

\author{
Jakkapan Khunnarong*, Siriwan Tangjitgamol, Sunamchok Srijaipracharoen
}

\begin{abstract}
Background: To evaluate the prevalence and features of other gynecologic or surgical lesions in endometrial cancer (EMC) patients. Materials and Methods: Clinico-pathological data of EMC patients who were treated in the institution from 1995 to 2012 were collected. Data collected were age, stage of disease according to the FIGO 2009 criteria (FIGO), histopathology, tumor grade, adjuvant therapy, other gynecologic or surgical lesions, follow-up period, and living status. Results: The mean age of 396 patients was $56.7 \pm 10.64$ years. Abnormal uterine bleeding was the most common presenting symptom $(90.1 \%)$. Bleeding was accompanied with pelvic mass in $7.7 \%$ and $5.4 \%$ had only a pelvic mass. Abnormal cervical cytology was found in 3.8\%.Approximately 75\% had early stage diseases and $86 \%$ had endometrioid histology. We found $55.8 \%$ of EMC patients had other gynecologic lesions: $89.6 \%$ benign and $9.5 \%$ malignant. Some $4.5 \%$ had pre-invasive cervical/vulva/vagina lesions. The two most common gynecologic lesions were myoma uteri and ovarian tumors. Focusing on the latter, approximately $14 \%$ were benign while $8 \%$ were malignant. Among 364 patients with available data, surgical lesions were found in $\mathbf{1 1 . 8 \%}, \mathbf{5 . 7 \%}$ benign and $9.2 \%$ malignant. The most common benign surgical condition was chronic appendicitis while breast and colon cancers were the two most common malignant lesions found. Conclusions: More than half of EMC patients had other gynecologic lesions including benign and malignant tumors. Surgical lesions were also found in more than one-tenth of patients. Careful pre-operative evaluation and intra-operative inspection are advised for proper management and better prognosis.
\end{abstract}

Keywords: Endometrial cancer - gynecologic lesions - surgical lesions

Asian Pac J Cancer Prev, 17 (2), 713-717

\section{Introduction}

The incidences of each female genital cancer vary among the continents. In the more developed regions, endometrial cancer (EMC) is the most common female genital tract malignancy with an estimated incidence of 13.0 per 100,000 women. The estimated incidence in less developed regions where cervical cancer is more prevalent, EMC is found less common of 5.9 per 100,000 in less developed regions or 4.3 per 100,000 women specifically in Thailand (Ferlay et al., 2013).

Mean age of the patients was approximately 63 years old for endometrioid and 67 years old for nonendometrioid (Howlader et al., 2013). The most common presenting symptom of EMC was abnormal uterine bleeding. Appropriate investigation to evaluate the cause of bleeding is mandatory before a proper management. However, some women may present with other complaints e.g. pelvic mass with or without symptom of bleeding. Previous studies from North America showed 0.4\%-0.6 $\%$ of EMC patients had no symptoms at all (Koss et al., 1981; Ouldamer et al., 2014). Without awareness, women in the latter group may undergo surgical treatment without recognition of underlying or associated EMC.
Primary treatment for EMC is total hysterectomy with bilateral salpingo-oophorectomy. Lymph node surgical evaluation is a subject of debate that it is an optional procedure to be selectively done in the patients with risk features or it should be universally executed. In a setting when the non-EMC lesions are the indication for surgery, EMC may be incidentally found during intra-operation or revealed from post-operative pathological diagnosis. In the first scenario when the operation is performed in a hospital where there is gynecologic oncology available, the intra-operative consultation could be done for an appropriate surgical management or she may have to be referred later for re-surgery or additional management if incomplete surgical staging is performed.

Most patients with EMC are found to have early stage diseases (stage I- II). This leads to a good prognosis with a 5-year overall survival rate of $96 \%$ for localized disease (Howlader et al., 2013). But in some situation, EMC were found during other gynecologic or surgical conditions. The previous study in Thailand reported $8.5 \%$ of EMC were discovered after surgery for benign gynecologic conditions (Srisomboon et al., 2001). This incidental finding led to incomplete surgical staging which might affect cancer survival. Furthermore, surgery of benign indications is 
Jakkapan Khunnarong et al

generally carried out by general surgeon or gynecologist. Data from a few studies reported worse treatment outcome when EMC was treated by general surgeons than the patients who had surgery performed by the gynecologic oncologist (Barnes and Kilgore, 2000; Chan et al., 2011).
Most studies of EMC came from the Western while there was limited report from Asia (Manchana and Khemapech 2008; Tangjitgamol et al., 2010; Binesh et al., 2014). There might be variations in risk factors and co-morbidities of EMC patients in other regions with

\section{Table 1. Clinico-pathologic Characteristic Features of Endometrial Cancer Patients}

\begin{tabular}{|c|c|c|}
\hline General characteristic features & $\mathrm{N}$ & $\%$ \\
\hline \multicolumn{3}{|l|}{ Gynecologic history $(\mathrm{n}=278)$} \\
\hline Nulliparous & 103 & 37.1 \\
\hline single & 73 & 26.3 \\
\hline married with infertility or without offspring & 30 & 10.8 \\
\hline Multiparous & 175 & 62.9 \\
\hline \multicolumn{3}{|l|}{ Symptoms $(n=336)$} \\
\hline Abnormal uterine bleeding & 303 & 90.1 \\
\hline Bleeding only & 277 & 82.4 \\
\hline Bleeding with pelvic mass & 26 & 7.7 \\
\hline Pelvic mass $b$ & 18 & 5.4 \\
\hline Abnormal Pap smear c & 15 & 4.5 \\
\hline \multicolumn{3}{|l|}{ Stage $(\mathrm{N}=396)$} \\
\hline I-II & 296 & 74.7 \\
\hline III-IV & 92 & 23.3 \\
\hline Unassigned stage (pre-operative radiation or chemotherapy) & 8 & 2 \\
\hline \multicolumn{3}{|l|}{ Histopathology $(\mathrm{N}=396)$} \\
\hline Endometrioid carcinoma with or without other minor components & 340 & 85.9 \\
\hline Others & 56 & 14.1 \\
\hline
\end{tabular}

Table 2. Other gynecologic lesions/ conditions in endometrial cancer patients ( $N=219$ patients)

\begin{tabular}{lrr}
\hline Other gynecologic conditions/ lesions & $\mathrm{n}$ & $\%$ \\
\hline Uterine lesions/ tumors & 167 & 42.2 \\
$\quad$ Leiomyoma, adenomyosis or adenomyoma & 11 & 2.8 \\
$\quad$ Endometrial polyps & 31 & 14.2 \\
Adnexal lesions/ tumors & 17 & 7.8 \\
$\quad$ Benign ovarian tumors & 7 & 1.8 \\
$\quad$ Malignant ovarian tumors & 11 & 2.8 \\
$\quad$ Partubal cyst or chronic salpingitis & 3 & 0.8 \\
Cervical lesions/ tumors & & \\
Pre-invasive or invasive cervical cancer a & 3 & \\
Pre-invasive or invasive vulvar or vaginal cancer b &
\end{tabular}

One patient may have multiple sites of lesions/ tumors. (b) Pre-invasive cervical lesions in nine and invasive cervical cancer in two patients. (c) Vaginal carcinoma, vulvar carcinoma, and vaginal intraepithelial neoplasia (one each)

Table 3. Surgical lesions/ conditions in endometrial cancer patients ( $\mathrm{N}=43$ patients)

\begin{tabular}{lrr}
\hline Other surgical conditions/ lesions & $\mathrm{n}$ & $\% \mathrm{a}$ \\
\hline Benign (n=17 patients with 21 lesions) & 2 & 5.1 \\
Breast mass/ nodules & 11 & 28.2 \\
Chronic appendicitis & 3 & 7.7 \\
Thyroid nodule/ mass & 3 & 7.7 \\
Colonic diverticulosis & 1 & 2.6 \\
Chronic cholecystitis & 1 & 2.6 \\
Focal hepatic nodular hyperplasia & & 30.8 \\
Malignant (n= 26 patients with 31 lesions) & 12 & 17.9 \\
Breast carcinoma b & 7 & 12.8 \\
Colon cancer & 5 & 5.1 \\
Lung cancer & 2 & 2.6 \\
Thyroid carcinoma c & 1 & 2.6 \\
Gall bladder carcinoma & 1 & 5.1 \\
Brain tumor & 2 & 2.6 \\
Skin cancer & 1 & 2.6 \\
Pancreatic carcinoma & 1 & \\
Appendiceal tumor & & \\
\hline
\end{tabular}

One patient may have more than one surgical lesions/ conditions. a Percentages obtained from the total number (N) of patients with surgical lesions/ conditions. (b) Three out of 11 patients with breast cancer also had cancers of colon (1), colon \& ovary (1), and skin (1). (c) One patient with thyroid cancer also had ovarian cancer 
different background on nutritional habits or genetic risks. We do not know whether EMC in our racial population would have different features than those from the other reports. With the lack of information, it is important that data of our own should be collected. We aimed to evaluate the associated gynecologic or surgical conditions in our Thai EMC patients.

\section{Materials and Methods}

We obtained an approval from the Ethics Committee for Research involving Human Subjects of the institution. Archives of the Gynecologic Oncology Unit of the Department of Obstetrics and Gynecology were searched to identify EMC patients treated between January 1995 and December 2012. We included the patients who had treatment in the institution and had endometrial carcinoma or carcinosarcoma. Exclusion criteria were patients who had other types of uterine sarcoma or had no available medical records.

Data collected were: age, presenting symptom, stage of disease according to the FIGO 2009 criteria (FIGO), histopathology, tumor grade, and adjuvant therapy. Other gynecologic or surgical lesions or conditions which may be recognized prior to, during or after EMC diagnosis and treatment were recorded.

Data were analyzed using SPSS statistical software, version 11.5 (SPSS, Chicago, IL). Descriptive statistics were used to analyze demographic data and were summarized as numbers with percentage or median with range. OS and PFS were analyzed by the Kaplan-Meier method and were compared between groups with log rank test. P-values of $<0.05$ were considered significant.

\section{Results}

We identified 446 EMC patients during the study period. Fifty were excluded for having other types of uterine sarcoma or had no medical records. Mean age of the 396 patients included in the study was $56.7 \pm 10.64$ years. Twenty-three patients $(5.8 \%)$ aged less than 40 years while $147(37.1 \%)$ aged $\geq 60$ years. Among 278 patients with available clinical data, 103 were nulliparous (37.1\%): being single in $73(26.3 \%)$ or having had history of infertile or never had a child in the other $30(10.8 \%)$. Eleven had tamoxifen as adjuvant therapy for their breast cancer while five had hormonal replacement therapy or herbal medicine for their menopausal symptoms. Of 336 patients with known complaining symptom, abnormal uterine bleeding was the most common found in 303 patients $(90.1 \%)$. Bleeding was accompanied with pelvic mass in 26 patients $(7.7 \%)$. Pelvic mass was the sole presenting symptom in 18 patients $(5.4 \%)$ and was the indication for surgery in almost all of them. Abnormal cervical cytology was abnormal in 15 patients (4.4\%), being: Papanicolaou class V in 13 and atypical glandular cells in the other two.

Regarding data of EMC, eight patients in our series $(2.0 \%)$ had either radiation or chemotherapy prior to surgery due to poor performance status to undergo extensive surgery for their advanced diseases. All other
DOI:http://dx.doi.org/10.7314/APJCP.2016.17.2.713 Other Gynecologic Pathology in Endometrial Cancer Patients 388 patients had primary surgical treatment. Six had radical, instead of simple, hysterectomy because there was presence of positive cancer tissue from endocervical curettage. Only one of them had stage II with cervical stroma invasion. The other five had stage I A or B (with cervical epithelial involvement in two). Early stage (stage I-stage II) EMC was more commonly found. The most common histopathology was endometrioid carcinoma with or without other minor components. Of 389 with available data, 172 patients (44.2\%) had adjuvant treatment after primary surgery. Characteristic features of the patients and data of EMC are shown in Table 1.

We identified 221 out of 396 patients $(55.8 \%)$ had other gynecologic lesions/ conditions while 43 out of 364 patients with available data (11.8\%) had surgical lesions/ conditions. There were no significant differences of ages among EMC patients who had or had no gynecologic or surgical lesions/ conditions (data not shown).

Among 221 EMC patients with gynecologic lesions, 198 patients had benign lesions $(89.6 \%)$ while 21 had malignant tumors $(9.5 \%)$ and ten had pre-invasive cervical or vulva/vagina lesions (4.5\%). Eight had benign tumors together with pre-invasive lesions or other cancers. All, except one prior cervical and two subsequent vaginal preinvasive and invasive vaginal cancer, other gynecologic lesions were co-incidental found during surgery for EMC. The two most common were myometrial and ovarian tumors. Table 2 shows details of other gynecologic lesions in EMC patients. Of 18 patients who had presenting symptom of pelvic mass, eight of which were myoma and/ or adenomyosis while the other eight were ovarian tumors (one benign and seven malignant) and only two were uterine enlargement from EMC. On the other hand, those 15 patients who had abnormal cervical cytology had no specific pathology of cervix.

Regarding the surgical lesions/ conditions in 43 patients, they were noted as benign in $17(5.7 \%)$ and malignant in 26 patients $(9.2 \%$ ) (Table 3). Among 26 patients who had malignant surgical conditions, four had more than one sites of non-gynecologic cancer: breast and colon cancer $(n=2)$, breast and non-melanoma skin cancer $(n=1)$, and colon cancer and carcinoid tumor of appendix $(n=1)$. Most of malignant surgical conditions were discovered prior to or after EM diagnosis while most benign surgical conditions were found at the time of EMC surgery.

\section{Discussion}

Treatment for EMC in more and less developed regions may differ based on the incidences, availability or access to health care service or health care policy for reimbursement, etc. In Thailand if EMC is recognized prior to surgery, the recommendation from the Thai Royal College of Obstetrics and Gynecology was to counsel the patients about the preference to have gynecologic oncologist be the surgeon to perform primary surgery unless there is minimal risk of extrauterine spread.

Our study had consistent finding with previous report that most of EMC patients presented with abnormal uterine bleeding (90\%) (Barakat et al., 1997). However, we found 
that nearly $18 \%$ had mixed symptoms of bleeding with pelvic mass or had pelvic mass alone, or abnormal cervical cytology. On the other hand, $10 \%$ of our EMC patients who had no uterine bleeding were comparable to $5 \%$ to $10 \%$ reported from previous study (Smith-Bindman et al., 1998). In a scenario when the patients presented with other symptoms or without symptoms, a misdiagnosis may lead to an inappropriate treatment. Other studies demonstrated $0.3 \%$ of EMC discovered in postmortem women who were dead from other causes (Horwitz et al., 1981) or very high range from $0.4 \%$ to $8.5 \%$ in women who underwent hysterectomy for benign gynecologic conditions (Srisomboon et al., 2001; Ouldamer et al., 2014). As found in our study that all patients who present with pelvic mass alone (5\%) underwent surgical exploration from this indication. These possibilities should be aware of particularly in a hospital setting where a complete or appropriate oncologic operation is not possible.

Our study demonstrated that up to $55 \%$ of EMC had other gynecologic lesions. Some of which had been existing while others were found coincidental or encountered after EMC. Most of the gynecologic lesions co-incidentally found in our study were uterine leiomyoma (42\%). This was much higher than 9\%-18\% of leiomyoma reported in general population (Zimmermann et al., 2012). This co-incidental pathology was not surprising because both EMC and uterine leiomyoma have common etiology of estrogen and progesterone milieu (Rein et al., 1995; Andersen 1996). However, the high incidence of leiomyoma in EMC in our study was higher than $29 \%$ in the study of Fortuny et al, which performed a case-control study in US population. The authors also reported a significant risk of patients with uterine leiomyoma to have EMC with odds ratio of 1.8 (Fortuny et al., 2009). Our high prevalence of uterine leiomyoma which was much higher than their study may be because our data were collected from pathological reports which recorded in detail any other findings aside from the main diagnosis while their study collected data through personal interviews.

Aside from benign tumor/ lesions, our study found more than $10 \%$ of our EMC patients had pre-invasive lesions and other non-EMC gynecologic cancer. Generally, epithelial ovarian cancer (EOC) was reported to be the most common synchronous cancer occurring in 5\% with EMC (Zaino et al., 2001). Our data showed slightly lower incidence with $4.2 \%$ of our EMC patients having synchronous EOC. Various incidences among studies might partly depend on the age of the EMC in each study because the synchronous EOC tended to occur higher in younger patients (Tangjitgamol et al., 2010). Regarding the extent of both cancers and their influences on treatment or outcomes, other studies also found that synchronous cancer tended to be in early stage and had good survival (Tangjitgamol et al., 2010; Lim et al., 2011). We found that slightly more than one third of our EMC patients who had EOC had more advanced OC than EMC. This might have required more extensive surgical procedures e.g. lymph node surgical resection or omentectomy. Any general surgeons or gynecologic oncologists who were going to do the operation for EMC should be cautious of this possibility to conduct an appropriate surgical procedure.
Aside other gynecologic tumors/ lesions, surgical lesions were found in almost $12 \%$ of our EMC patients. They might be discovered prior to, during, or post EMC treatment. Focus on malignant tumors, the most common cancer that we found with EOC was breast follow by colon and lung cancers in respective orders. The detail of other cancers would be described and presented in our other works. Focusing on benign surgical tumors/ lesions, most of them would not impact the line of treatment for EMC except for one patient who had focal hepatic nodular hyperplasia found at the time of surgical staging for EMC. If intra-operative consultation to a surgeon was not possible, she might need another surgery or it might be interpreted as metastatic tumor from EMC requiring adjuvant treatment.

In conclusion, Our study demonstrated high coincidence of other gynecologic or surgical pathology in EMC patients. The most common of these lesion/ tumors was uterine leiomyoma. The surgeons who plan to operate for benign gynecologic/surgical lesions especially leiomyoma should perform an appropriate pre-operative or intra-operative evaluation for a possible co-incidence of EMC.

\section{References}

Andersen J (1996). Growth factors and cytokines in uterine leiomyomas. Semin Reprod Endocrinol, 14, 269-82.

Barakat RR, Park RC, Grigsby PW, et al (1997). Corpus: epithelial tumors. In: Hoskins WJ, Perez CA, Young RC, editors. Principles and practice of gynecologic oncology. $2^{\text {nd }}$ ed. Philadelphia: Lippincott-Raven, 859-96.

Barnes MN, Kilgore LC (2000). Complete surgical staging of early endometrial adenocarcinoma: optimizing patient outcomes. Semin Radiat Oncol, 10, 3-7.

Binesh F, Akhavan A, Behniafard N, et al (2014). Endometrial adenocarcinoma: clinicopathologic and survival characteristics in Yazd, Iran. Asian Pac J Cancer Prev, 15, 2797-801.

Chan JK, Sherman AE, Kapp DS, et al (2011). Influence of gynecologic oncologists on the survival of patients with endometrial cancer. J Clin Oncol, 29, 832-8.

Ferlay J, Soerjomataram I, Ervik M, et al (2013). GLOBOCAN 2012 v1.0, Cancer. Incidence and Mortality Worldwide: IARC Cancer Base No. 11 [Internet]. Lyon, france: international agency for research on cancer.

Fortuny J, Sima C, Bayuga S, et al (2009). Risk of endometrial cancer in relation to medical conditions and medication use. Cancer Epidemiol Biomarkers Prev, 18, 1448-56.

Horwitz RI, Horwitz SM, Feinstein R, et al (1981). Necropsy diagnosis of endometrial cancer and detection-bias in case/ control studies. Lancet, 2, 66-8.

Howlader N, Noone AM, Krapcho M, et al (2013). SEER cancer statistics review, 1975-2011 based on November 2013 SEER data submission. National Cancer Institute. Bethesda, MD.

Koss L, Schreiber K, Oberlander S, et al (1981). Screening of asymptomatic women for endometrial cancer. CA Cancer J Clin, 31, 300-17.

Lim YK, Padma R, Foo L, et al (2011). Survival outcome of women with synchronous cancers of endometrium and ovary: a 10 year retrospective cohort study. J Gynecol Oncol, 22, 239-43.

Manchana T, Khemapech N (2008). Endometrial adenocarcinoma in young Thai women. Asian Pac J Cancer Prev, 9, 283-6.

Ouldamer L, Rossard L, Arbion F, et al (2014). Risk of incidental 
finding of endometrial cancer at the time of hysterectomy for benign condition. J Minim Inv Gynecol, 21, 131-5.

Rein MS, Barbieri RL, Friedman AJ (1995). Progesterone: a critical role in the pathogenesis of uterine myomas. Am J Obstet Gynecol, 172, 14-8.

Smith-Bindman R, Kerlikowske K, Feldstein V, et al (1998). Endovaginal ultrasound to exclude endometrial cancer and other endometrial abnormalities. JAMA, 280, 1510-7.

Srisomboon J, Phongnarisorn C, Suprasert P (2001). Endometrial cancer diagnosed in patients undergoing hysterectomy for benign gynecologic conditions. Thai J Obstet Gynaecol, 13, 29-32.

Tangjitgamol S, Manusirivithaya S, Srijaipracharoen S, et al (2010). Endometrial cancer in thai women: clinicopathological presentation and survival. Asian Pacific $J$ Cancer Prev, 11, 1267-72.

Zaino R, Whitney C, Brady MF, et al (2001). Simultaneously detected endometrial and ovarian carcinomas: a prospective clinicopathologic study of 74 cases: a gynecologic oncology group study. Gynecol Oncol, 83, 355-62.

Zimmermann A, Bernuit D, Gerlinger C, et al (2012). Prevalence, symptoms and management of uterine fibroids: an international internet-based survey of 21,746 women. $B M C$ Women's Health, 12, 6. 